Research Article

\title{
Non-semiregular bipartite graphs with integer Sombor index
}

\author{
Mohammad Reza Oboudi* \\ Department of Mathematics, College of Sciences, Shiraz University, Shiraz, 71457-44776, Iran
}

(Received: 4 October 2021. Received in revised form: 16 October 2021. Accepted: 16 October 2021. Published online: 23 October 2021.)

(C) 2021 the author. This is an open access article under the CC BY (International 4.0) license (www.creativecommons.org/licenses/by/4.0/).

\begin{abstract}
For a simple graph $G$, the Sombor index (a recently introduced vertex-degree based molecular structure descriptor) is defined as $S O(G)=\sum_{u v \in E(G)} \sqrt{d_{u}^{2}+d_{v}^{2}}$, where $d_{v}$ is the degree of $v$. A graph is bipartite semi-regular if it is bipartite bidegreed and all vertices in the same class of bipartition have the same degree. In the recent paper [T. Došlić, T. Réti, A. Ali, Discrete Math. Lett. 7 (2021) 1-4] the following claim was posed: "Let $G$ be a connected bipartite graph. Then $S O(G)$ is an integer if and only if $G$ is bipartite semi-regular and its degrees $\delta$ and $\Delta$ appear as non-maximal elements in some Pythagorean triple". In the present paper we show that the 'only if' part of the mentioned claim is not true. More precisely, we construct infinite number of connected bipartite graphs such that in their degree sequences there are three or four distinct numbers.
\end{abstract}

Keywords: Sombor index of graphs; semiregular graphs; bipartite graphs.

2020 Mathematics Subject Classification: 05C07, 05C09.

\section{Introduction}

Throughout this paper all graphs are simple, that is they are finite and undirected, without loops and multiple edges. Let $G=(V(G), E(G))$ be a simple graph. The order of $G$ is the number of vertices of $G$. By $e=u v$ we mean the edge $e$ between $u$ and $v$. For a vertex $v \in V(G)$, the degree of $v$ is the number of edges incident with $v$ and is denoted by $\operatorname{deg}_{G}(v)$. A pendant vertex is a vertex with degree one and a pendant edge is an edge such that one of its end vertices is pendant vertex. A $k$-regular graph is a graph such that every vertex has degree $k$. The set of all different degrees of the vertices of a graph $G$ is known as the degree set of $G$. A graph whose degree set consists of one/two/three element(s) is called regular/bidegreed/tridegreed graph, respectively. A graph is bipartite semiregular if it is bipartite bidegreed and all vertices in the same class of bipartition have the same degree. Thus in these graphs all edges connect vertices of different degrees. The edgeless graph, the complete graph, the cycle, and the path of order $n$, are denoted by $\overline{K_{n}}, K_{n}, C_{n}$ and $P_{n}$, respectively. Let $t$ and $n_{1}, \ldots, n_{t}$ be some positive integers. By $K_{n_{1}, \ldots, n_{t}}$ we mean the complete multipartite graph with parts size $n_{1}, \ldots, n_{t}$. In particular, the complete bipartite graph with part sizes $m$ and $n$ denoted by $K_{m, n}$. The star of order $n$, denoted by $S_{n}$, is the complete bipartite graph $K_{1, n-1}$. The open neighborhood of a vertex $v$ of a graph $G$, denoted by $N_{G}(v)$, is the set of all neighbors of $v$ in $G$. For every subset of vertices (edges) of $G$, say $L$, by $G \backslash L$ we mean the graph that is obtained from $G$ by removing the vertices of $L$ (the edges of $L$ ). If $u$ is a vertex (an edge) of $G$, we use $G \backslash v$ instead of $G \backslash\{v\}$. For two vertices $u$ and $v$ in a connected graph $G$, the distance between $u$ and $v$, denoted by $d_{G}(u, v)$, is the length of a shortest path between them. The diameter of $G$, denoted by $\operatorname{diam}(G)$, is the greatest distance between any pair of its vertices.

In chemical graph theory there are many indices (topological indices). Recently, a new index, Sombor index, has been introduced by Ivan Gutman in [4]. For a graph $G$, the Sombor index of $G$, denoted by $S O(G)$, is defined as

$$
S O(G)=\sum_{u v \in E(G)} \sqrt{d_{u}^{2}+d_{v}^{2}}
$$

where $d_{v}$ is the degree of $v$. For example the Sombor index of the star $S_{n}$ is $(n-1) \sqrt{(n-1)^{2}+1}$. There are many papers related to Sombor index, for instance see [1-6] and the references therein. One of the interesting subject related to Sombor index is studying the graphs such that their Sombor index are integer. In [3] and [5] the authors obtain some results related to this problem. In [3] the following proposition was posed:

Proposition 1.1. Let $G$ be a connected bipartite graph. Then $S O(G)$ is an integer if and only if $G$ is bipartite semiregular and its degrees $\delta$ and $\Delta$ appear as non-maximal elements in some Pythagorean triple.

*E-mail address: mr_oboudi@yahoo.com,mr_oboudi@shirazu.ac.ir 
In this paper we show that the 'only if' part of Proposition 1.1 is not true. In fact we construct infinite number of connected bipartite graphs such that in their degree sequence there are three or four distinct numbers.

\section{Non-semiregular bipartite graphs}

In this section we study the connected non-semiregular bipartite graphs with integer Sombor index. For a positive integer $t \geq 1$, by a $t$-degree graph we mean a graph such that its degree sequence has exactly $t$ distinct numbers. In particular, 1-degree graphs are regular graphs, and 2-degree graphs are bidegreed graphs. Let $G$ be a graph with vertex set $\left\{v_{1}, \ldots, v_{n}\right\}$. Assume that $H_{1}, \ldots, H_{n}$ are some disjoint graphs. By $G\left[H_{1}, \ldots, H_{n}\right]$ we mean the graph that is obtained by replacing the vertex $v_{j}$ by the graph $H_{j}$, for $1 \leq j \leq n$, such that every vertex of $H_{i}$ is adjacent to every vertex of $H_{j}$ if and only if $v_{i}$ is adjacent to $v_{j}$ in $G$. For example $P_{2}\left[K_{p}, K_{q}\right]$ is the complete graph $K_{p+q}$ and $P_{2}\left[\overline{K_{p}}, \overline{K_{q}}\right]$ is the complete bipartite graph $K_{p, q}$. We note that $G\left[\overline{K_{m_{1}}}, \ldots, \overline{K_{m_{n}}}\right]$ is bipartite if and only if $G$ is bipartite. Here we construct some $t$-degree graphs with integer Sombor index for $t \in\{3,4\}$. It is not hard to check the following results.

Theorem 2.1. Assume that $a>b>c \geq 1$ are some positive integers such that $a^{2}+b^{2}, a^{2}+c^{2}$ and $b^{2}+c^{2}$ are square. Let $P$ be the path of order 4 with the vertex set $\left\{v_{1}, v_{2}, v_{3}, v_{4}\right\}$ and the edge set $\left\{v_{1} v_{2}, v_{2} v_{3}, v_{3} v_{4}\right\}$. Let $G(a, b, c)=P\left[\overline{K_{a-c}}, \overline{K_{c}}, \overline{K_{c}}, \overline{K_{b-c}}\right]$. Then the order of $G(a, b, c)$ is $a+b, G(a, b, c)$ is a connected bipartite 3-degree graph with vertex degrees $a, b, c$ and with integer Sombor index. In fact,

$$
S O(G(a, b, c))=c(b-c) \sqrt{b^{2}+c^{2}}+c^{2} \sqrt{a^{2}+b^{2}}+c(a-c) \sqrt{a^{2}+c^{2}} .
$$

Remark 2.1. Suppose that $a>b>c \geq 1$ are some positive integers such that $a^{2}+b^{2}, a^{2}+c^{2}$ and $b^{2}+c^{2}$ are square. Using computer we find that if $a \leq 1000$, then $(a, b, c)$ is one of the following

$$
\begin{aligned}
& (240,117,44),(275,252,240),(480,234,88),(550,504,480),(693,480,140), \\
& (720,132,85),(720,351,132),(792,231,160),(825,756,720),(960,468,176) .
\end{aligned}
$$

Using Theorem 2.1 and one of the triples that has been mentioned in Remark 2.1 (for example using triple $(240,117,44)$ ) we find infinite number of connected 3-degree bipartite graph with integer Sombor index.

Corollary 2.1. Let $P$ be the path of order 4 with the vertex set $\left\{v_{1}, v_{2}, v_{3}, v_{4}\right\}$ and the edge set $\left\{v_{1} v_{2}, v_{2} v_{3}, v_{3} v_{4}\right\}$. Then for every integer $m \geq 1$, the graph $P\left[\overline{K_{196 m}}, \overline{K_{44 m}}, \overline{K_{44 m}}, \overline{K_{73 m}}\right]$ has integer Sobmor index. More precisely,

$$
S O\left(P\left[\overline{K_{196 m}}, \overline{K_{44 m}}, \overline{K_{44 m}}, \overline{K_{73 m}}\right]\right)=44 m(73 m)(125 m)+1936 m^{2}(267 m)+44 m(196 m)(244 m),
$$

that is

$$
S O\left(P\left[\overline{K_{196 m}}, \overline{K_{44 m}}, \overline{K_{44 m}}, \overline{K_{73 m}}\right]\right)=3022668 m^{3} .
$$

Theorem 2.2. Assume that $a>b>c>d \geq 1$ are some positive integers such that $a^{2}+c^{2}, a^{2}+d^{2}$ and $b^{2}+c^{2}$ are square. Let $H$ be the graph with vertex set $V(H)=\left\{v_{1}, \ldots, v_{5}\right\}$ and the edge set $E(H)=\left\{v_{1} v_{2}, v_{2} v_{3}, v_{3} v_{4}, v_{4} v_{1}, v_{4} v_{5}\right\}$. In fact $H$ is obtained by adding a pendant edge to a vertex of the cycle $C_{4}$. Let $G(a, b, c, d)=H\left[\overline{K_{1}}, \overline{K_{c-d}}, \overline{K_{b-1}}, \overline{K_{d}}, \overline{K_{a-b}}\right]$. Then the order of $G(a, b, c, d)$ is a $+c, G(a, b, c)$ is a connected bipartite 4-degree graph with vertex degrees $a, b, c, d$ and with integer Sombor index. In fact,

$$
S O(G(a, b, c, d))=(c-d) \sqrt{b^{2}+c^{2}}+d \sqrt{a^{2}+c^{2}}+(b-1)(c-d) \sqrt{b^{2}+c^{2}}+d(b-1) \sqrt{a^{2}+c^{2}}+d(a-b) \sqrt{a^{2}+d^{2}} .
$$

Remark 2.2. Suppose that $a>b>c>d \geq 1$ are some positive integers such that $a^{2}+c^{2}, a^{2}+d^{2}$ and $b^{2}+c^{2}$ are square. Using computer we find that if $a \leq 160$, then $(a, b, c, d)$ is one of the following

$$
(48,21,20,14),(72,40,30,21),(80,52,39,18),(80,63,60,18),(80,63,60,39),
$$

$(96,42,40,28),(96,75,40,28),(105,90,56,36),(112,88,66,15),(120,36,27,22)$,

$(120,84,35,22),(120,84,35,27),(135,112,84,72),(140,68,51,48),(144,56,42,17)$,

$(144,63,60,17),(144,63,60,42),(144,80,60,17),(144,80,60,42),(144,91,60,17)$,

$(144,91,60,42),(160,104,78,36),(160,126,120,36),(160,126,120,78)$.

Now by using Theorem 2.2 and one of the quadruples that has been mentioned in Remark 2.2 (for example using quadruple $(160,126,120,36))$ we find infinite number of connected 4-degree bipartite graph with integer Sombor index. 
Corollary 2.2. Let $H$ be the graph with vertex set $V(H)=\left\{v_{1}, \ldots, v_{5}\right\}$ and the edge set $E(H)=\left\{v_{1} v_{2}, v_{2} v_{3}, v_{3} v_{4}, v_{4} v_{1}, v_{4} v_{5}\right\}$. Thus $H$ is obtained by adding a pendant edge to a vertex of the cycle $C_{4}$. Then for every integer $m \geq 1$, the graph $H\left[\overline{K_{m}}, \overline{K_{84 m}}, \overline{K_{125 m}}, \overline{K_{36 m}}, \overline{K_{34 m}}\right]$ has integer Sobmor index. More precisely,

$$
\begin{aligned}
& S O\left(H\left[\overline{K_{m}}, \overline{K_{84 m}}, \overline{K_{125 m}}, \overline{K_{36 m}}, \overline{K_{34 m}}\right]\right)=84 m(174 m)+36 m(200 m)+ \\
& (126 m-1)(84 m)(174 m)+36 m(126 m-1)(200 m)+36 m(34 m)(164 m) .
\end{aligned}
$$

Thus

$$
S O\left(H\left[\overline{K_{m}}, \overline{K_{84 m}}, \overline{K_{125 m}}, \overline{K_{36 m}}, \overline{K_{34 m}}\right]\right)=2949552 m^{3} .
$$

\section{Acknowledgment}

The author is grateful to the referees for their helpful comments.

\section{References}

[1] R. Cruz, I. Gutman, J. Rada, Sombor index of chemical graphs, Appl. Math. Comput. 399 (2021) \#126018.

[2] K. C. Das, A. S. Cevik, I. N. Cangul, Y. Shang, On Sombor index, Symmetry 13 (2021) \#140.

[3] T. Došlić, T. Réti, A. Ali, On the structure of graphs with integer Sombor indices, Discrete Math. Lett. 7 (2021) 1-4.

[4] I. Gutman, Geometric Approach to Degree-Based Topological Indices: Sombor Indices, MATCH Commun. Math. Comput. Chem. 86 (2021) 11-16.

[5] T. Réti, T. Došlić, A. Ali, On the Sombor index of graphs, Contrib. Math. 3 (2021) 11-18.

[6] T. Zhou, Z. Lin, L. Miao, The Sombor index of trees and unicyclic graphs with given maximum degree, Discrete Math. Lett. 7 (2021) 24-29. 